\title{
Metalloprotein Oxidation States Spatially Resolved by Anomalous Dispersion Crystallography
}

Nicholas K. Sauter ${ }^{1}$, James Holton ${ }^{1}$, Jan Kern ${ }^{1}$, Junko Yano ${ }^{1}$, Vittal

Yachandra $^{1}$

${ }^{1}$ Lawrence Berkeley National Laboratory, Berkeley, CA, USA

Using serial femtosecond crystallography we will investigate the electronic environments at individual metal centers in metalloproteins by observing diffraction from a wavelength scan through an absorption edge. Other groups have applied such scans to single crystals at synchrotron sources; e.g., the assignment of oxidation states to Fe atoms in mixedvalence (Fe(II)/Fe(III)) nitrogenase [Spatzal et al. (2016) Nat. Commun. 7, 10902]. With the collection of diffraction datasets at multiple wavelengths, the Bijvoet (samewavelength) and dispersive (between-wavelength) differences will yield separate scattering factors for each metal site and at each energy. The wavelength-dependence of the absorption coefficient, f-double-prime, is a sensitive indicator of the electronic state, with the absorption peak shifted to lower energies for more reduced states. XFEL crystallography offers three breakthrough advantages for this method: 1) the femtosecond time scale avoids X-ray photoreduction at specific metal centers, 2) the electronic environment can be probed under biological conditions, rather than the cryo-temperatures used at synchrotrons, and $3)$ it is possible to probe the time domain. For metalloproteins with multiple metal centers that are difficult to differentiate by conventional spectroscopy (such as the four-Mn oxygen-evolving complex of photosystem II, a future target), diffraction-detected absorption spectra thus offer a unique window on catalytic mechanisms. Here we present simulated diffraction data that establish our data processing methods in principle, as well as preliminary experimental data collected at LCLS.

Work was supported by NIH grants GM117126 and GM110501. 